\title{
Effect of impurity atoms on the adsorption/dissociation of hydrogen sulfide and hydrogen diffusion on $\mathrm{Fe}(100)$ surface
}

Jingxuan Liang a , Xiangli Wen a, b, Shikai Wei a , Shuqi Zheng a, *

${ }^{\text {a }}$ State Key Laboratory of Heavy Oil Processing, Department of Materials Science and Engineering, China University of Petroleum (Beijing), Beijing, 102249, PR China.

${ }^{\mathrm{b}}$ State Key Laboratory of Tribology, Department of Mechanical Engineering, Tsinghua University, Beijing 100084, PR China.

* Corresponding author. Tel.: +86 0108973 3200; Fax: +86 01089733200

E-mail address: zhengsq09@163.com. 


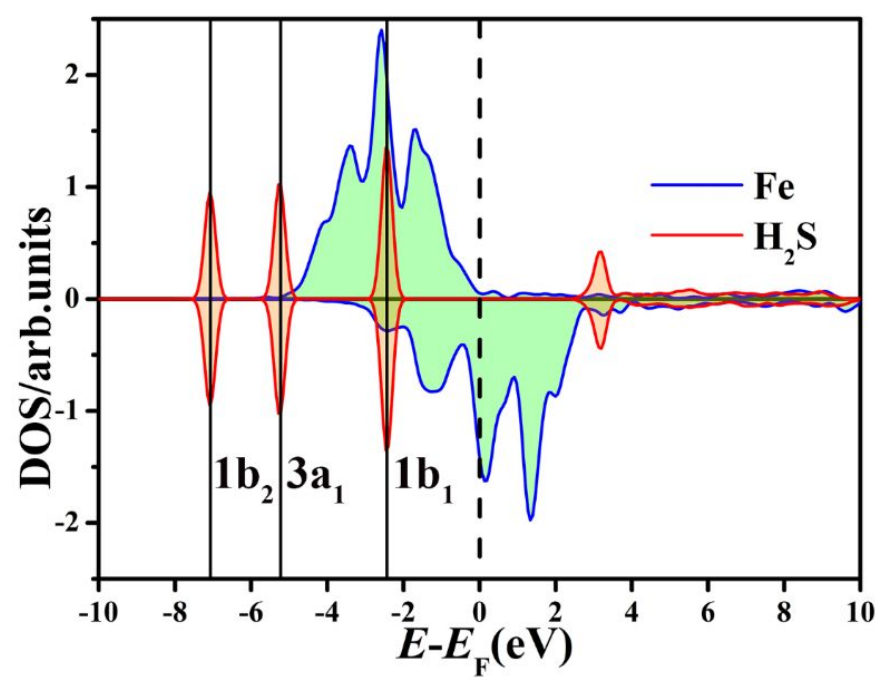

Figure S1. PDOS of the isolated $\mathrm{H}_{2} \mathrm{~S}$ and free surface slab model.
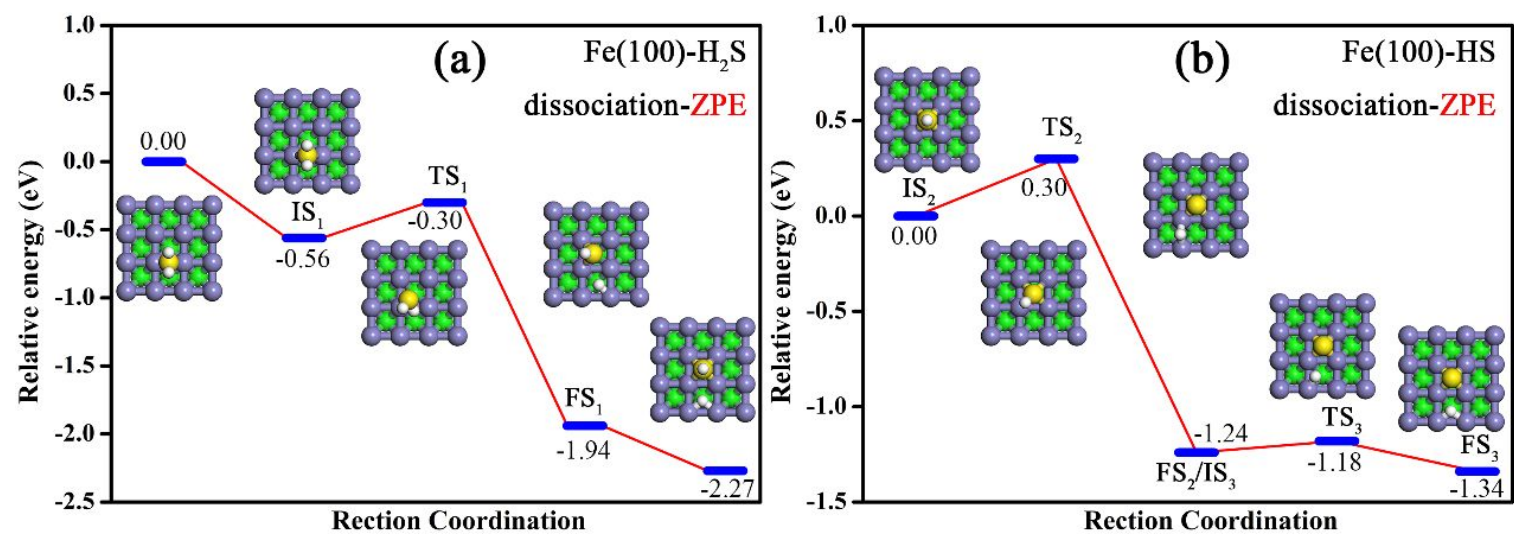

Figure S2. Top view of the intermediate state and the transition state of most favorable path for the dissociation of $\mathrm{H}_{2} \mathrm{~S}$ (a) and $\mathrm{HS}$ (b) on the pristine $\mathrm{Fe}(100)$ surface, including ZPE correction.

Table S1. The adsorption energy, adsorption sites and structural parameters of HS, H $+\mathrm{HS}$ and $\mathrm{H}+\mathrm{S}$ adsorbed on different $\mathrm{Fe}(100)$ surfaces.

\begin{tabular}{ccccc}
\hline Adsorption atom & Adsorption site & $d(\AA)$ & $d(\mathrm{~S}-\mathrm{H})(\AA)$ & $E_{\text {ads }}(\mathrm{eV})$ \\
\hline \multirow{4}{*}{$\mathrm{H}+\mathrm{HS}$} & $\mathrm{H}+\mathrm{H}$ & $1.878 ; 2.339$ & 1.378 & -2.18 \\
$\mathrm{HS}$ & $\mathrm{H}$ & 2.343 & 1.385 & -3.86 \\
$\mathrm{H}+\mathrm{S}$ & $\mathrm{H}+\mathrm{H}$ & $1.830 ; 2.342$ & ---- & -5.17 \\
& \multicolumn{4}{c}{$\mathrm{Fe}(100)-\mathrm{H}$} \\
$\mathrm{H}+\mathrm{HS}$ & $\mathrm{H}+\mathrm{H}$ & $1.909 ; 2.344$ & 1.388 & $-\mathbf{- 2 . 2 5}$ \\
& $\mathrm{H}+\mathrm{B}$ & $1.858 ; 2.319$ & 1.360 & -1.87 \\
& $\mathrm{~B}+\mathrm{B}$ & $1.698 ; 2.324$ & 1.363 & -1.78 \\
& $\mathrm{~B}+\mathrm{H}$ & $1.695 ; 2.346$ & 1.386 & -2.15
\end{tabular}




\begin{tabular}{|c|c|c|c|c|}
\hline \multirow[t]{2}{*}{ HS } & $\mathrm{H}$ & 2.345 & 1.387 & -3.86 \\
\hline & B & 2.326 & 1.368 & -3.48 \\
\hline \multirow[t]{3}{*}{$\mathrm{H}+\mathrm{S}$} & $\mathrm{H}+\mathrm{H}$ & $1.889 ; 2.319$ & ---- & -5.18 \\
\hline & $\mathrm{B}+\mathrm{H}$ & $1.691 ; 2.315$ & ---- & -5.08 \\
\hline & \multicolumn{4}{|c|}{$\mathrm{Fe}(100)-\mathrm{O}$} \\
\hline \multirow[t]{5}{*}{$\mathrm{H}+\mathrm{HS}$} & $\mathrm{H}+\mathrm{H}$ & $1.847 ; 2.344$ & 1.388 & -2.15 \\
\hline & $\mathrm{H}+\mathrm{B}$ & $1.877 ; 2.312$ & 1.361 & -1.77 \\
\hline & $\mathrm{B}+\mathrm{B}$ & $1.699 ; 2.322$ & 1.361 & -1.70 \\
\hline & $\mathrm{B}+\mathrm{H}$ & $1.694 ; 2.348$ & 1.384 & -2.11 \\
\hline & $\mathrm{C}^{\mathrm{a}}+\mathrm{H}$ & $2.838 ; 2.360$ & 1.386 & -1.54 \\
\hline \multirow[t]{2}{*}{$\mathrm{HS}$} & $\mathrm{H}$ & 2.345 & 1.386 & -3.82 \\
\hline & B & 2,328 & 1.362 & -3.42 \\
\hline \multirow[t]{2}{*}{$\mathrm{H}+\mathrm{S}$} & $\mathrm{H}+\mathrm{H}$ & $1.810 ; 2.319$ & ---- & -5.10 \\
\hline & $\mathrm{B}+\mathrm{H}$ & $1.696 ; 2.314$ & ---- & -5.06 \\
\hline \multicolumn{5}{|c|}{$\mathrm{Fe}(100)-\mathrm{S}$} \\
\hline \multirow[t]{3}{*}{$\mathrm{H}+\mathrm{HS}$} & $\mathrm{H}+\mathrm{H}$ & $1.819 ; 2.329$ & 1.383 & -2.16 \\
\hline & $\mathrm{B}+\mathrm{H}$ & $1.688 ; 2.340$ & 1.385 & -2.14 \\
\hline & $\mathrm{C}+\mathrm{H}$ & $3.288 ; 2.341$ & 1.384 & -0.89 \\
\hline HS & $\mathrm{H}$ & 2.332 & 1.383 & -3.86 \\
\hline \multirow{2}{*}{$\mathrm{H}+\mathrm{S}$} & $\mathrm{H}+\mathrm{H}$ & $1.799 ; 2.309$ & ---- & -5.05 \\
\hline & $\mathrm{B}+\mathrm{H}$ & $1.688 ; 2.300$ & ---- & -5.02 \\
\hline
\end{tabular}

${ }^{\mathrm{a}} \mathrm{C}$ : represents $\mathrm{H}$ atom and impurity atom combine to form $\mathrm{OH}$ or $\mathrm{HS}$. 